\title{
A SIMPLE AND EFFICIENT SYNTHESIS OF SUBSTITUTED PYRAZOLES
}

\section{Doddamani Shridevi Doddaramappa ${ }^{1}$, Chandra $^{2}$,}

\author{
M. Mahendra ${ }^{2}$, and K. M. Lokanatha Rai ${ }^{1 *}$
}

A novel route for the formation of 3-aryl-1-phenyl-1H-pyrazoles was discovered upon the reaction of 3-aryl-1-phenyl-4,5-dihydro-1H-pyrazole-5-carbonitrile with potassium hydroxide in the presence of a phase transfer catalyst (tetrabutylammonium bromide) in THF as a solvent, and a single crystal X-ray study is reported.

Keywords: 1,3-diphenyl-1H-pyrazole, potassium hydroxide, pyrazole-5-carbonitrile, phase transfer catalyst, tetrabutylammonium bromide, X-ray structural study.

There is a large number of organic chemicals that are synthesized by reacting two immiscible reactants present in two immiscible phases. Under such conditions, the reaction is carried out using a phase transfer catalyst. Phase transfer catalysis (PTC) has been applied to over 600 industrial processes to produce a variety of chemicals, such as intermediates, dyestuffs, agrochemicals, perfumes, flavors, pharmaceuticals, and polymers [13]. Pyrazoles are popular targets for synthetic chemists, primarily because of their diverse and useful biological properties [4]. They are, therefore, attractive building blocks for pharmaceutical research and are present as structural motifs in leading pharmaceuticals, including blockbuster drugs such as Celebrex [5] and Viagra [6]. A large number of pyrazole derivatives exhibit a wide spectrum of biological activities and are used as antiviral, antitumor, antiinflammatory, antimicrobial, cholesterol-lowering, antidepressant, and antipsychotic agents [7-12].

Due to the important applications of pyrazoles, their synthesis has been extensively studied [13-20]. So far, the main route to fully functionalized pyrazoles involves condensation reactions between hydrazines and 1,3-dicarbonyl compounds [21-23] or alkyne derivatives [24-25] and 1,3-dipolar cycloadditions [26-27]. 1,3-Diphenyl-1H-pyrazole was synthesized by the condensation of $\mathrm{C}(\alpha)$-anions or trianions with esters [28]. In 1983, Doyle and co-workers synthesized pyrazoles from cyanopyrazolines under basic conditions [29]. Farag and co-workers have synthesized polysubstituted pyrazole derivatives via 1,3-dipolar cycloaddition reactions [30]. Zohdi and co-workers have also synthesized pyrazoles by reaction of hydrazonoyl chlorides with 2-aryl1-cyano-1-thiazol-2-ylethenes in the presence of triethylamine to give cycloadducts, that were converted to the corresponding pyrazoles by the action of sodium methoxide [31]. Attaryan et al. have synthesized 1-(2-amino-

\footnotetext{
*To whom correspondence should be addressed, e-mail: kmlrai@yahoo.com.

${ }^{1}$ University of Mysore, Manasagangotri, Mysore-570006, India.

${ }^{2}$ Department of Studies in Physics, University of Mysore, Manasagangotri, Mysore-570006, India; e-mail: mychandru.10@gmail.com, mahendra@physics.uni-mysore.ac.in.
}

Published in Khimiya Geterotsiklicheskikh Soedinenii, No. 5, pp. 748-753, May, 2013. Original article submitted January 29, 2013; revision submitted April 21, 2013. 
thyl)pyrazoles with 2-chloroethylamine under phase transfer catalysis [32]. In view of the above findings, it was considered worthwhile to describe the synthesis using PTC and crystal studies of 1,3-diphenyl-1H-pyrazole derivatives.

Herein we report for the first time a mild and rapid conversion of 3-aryl-1-phenyl-4,5-dihydro$1 H$-pyrazole-5-carbonitrile to 3-aryl-1-phenyl-1H-pyrazole in high yield using a phase transfer catalyst. The starting materials, aromatic aldehyde hydrazones $\mathbf{1 a - h}$, were prepared from the corresponding aldehydes employing known methods [33]. Oxidative dehydrogenation of hydrazones 1a-h using chloramine-T afforded nitrile imines which were intercepted in situ by acrylonitrile in refluxing ethanol to yield 3-aryl-1-phenyl-4,5-dihydro-1H-pyrazole-5-carbonitriles 2a-h [34]. During our present study we observed that the treatment of pyrazole carbonitriles 2a-h with potassium hydroxide and tetrabutylammonium bromide (TBAB) in THF provided the corresponding 3-aryl-1-phenyl-1 $H$-pyrazoles 3a-h in good yield (Table 1).

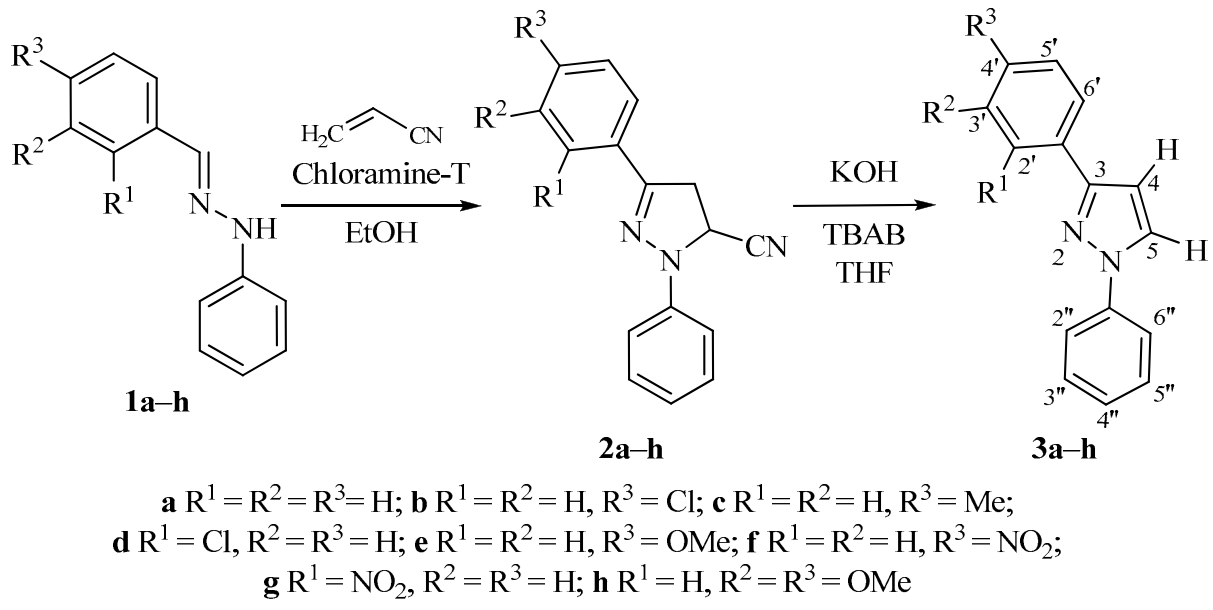

We found that the optimal reaction protocol was to add potassium hydroxide and tetrabutylammonium bromide to a solution of the pyrazole $\mathbf{2 a}$ in a minimum volume of THF at room temperature. Once the reaction was completed, the reaction mass was concentrated under reduced pressure, extracted with ether, washed with water, and dried over anhydrous sodium sulfate. Using this protocol, the 1,3-diphenyl-1H-pyrazole (3a) was isolated in $95 \%$ yield. In an attempt to see whether these conditions were applicable to a broader range of starting materials, reactions of various substituted pyrazoles $\mathbf{2} \mathbf{b}-\mathbf{h}$ under similar conditions were examined.

TABLE 1. Physicochemical Characteristics of Substituted Pyrazoles 3a-h

\begin{tabular}{|c|c|c|c|c|c|c|}
\hline \multirow{2}{*}{$\begin{array}{l}\text { Com- } \\
\text { pound }\end{array}$} & \multirow{2}{*}{$\begin{array}{l}\text { Empirical } \\
\text { formula }\end{array}$} & \multicolumn{3}{|c|}{$\begin{array}{c}\text { Found, } \% \\
\text { Calculated, } \%\end{array}$} & \multirow[t]{2}{*}{$\mathrm{Mp},{ }^{\circ} \mathrm{C}$} & \multirow[t]{2}{*}{ Yield, \% } \\
\hline & & $\mathrm{C}$ & $\mathrm{H}$ & $\mathrm{N}$ & & \\
\hline $3 \mathbf{a}$ & $\mathrm{C}_{15} \mathrm{H}_{12} \mathrm{~N}_{2}$ & $\frac{81.60}{81.79}$ & $\frac{5.50}{5.49}$ & $\frac{12.80}{12.72}$ & $80-82$ & 95 \\
\hline $3 \mathbf{b}$ & $\mathrm{C}_{15} \mathrm{H}_{11} \mathrm{ClN}_{2}$ & $\frac{70.72}{70.73}$ & $\frac{4.32}{4.35}$ & $\frac{11.01}{11.00}$ & $117-119$ & 94 \\
\hline $3 c$ & $\mathrm{C}_{16} \mathrm{H}_{14} \mathrm{~N}_{2}$ & $\frac{82.01}{82.02}$ & $\frac{6.01}{6.02}$ & $\frac{11.80}{11.96}$ & $122-124$ & 88 \\
\hline 3d & $\mathrm{C}_{15} \mathrm{H}_{11} \mathrm{ClN}_{2}$ & $\frac{70.70}{70.73}$ & $\frac{4.31}{4.35}$ & $\frac{11.02}{11.00}$ & $135-137$ & 90 \\
\hline $3 e$ & $\mathrm{C}_{16} \mathrm{H}_{14} \mathrm{~N}_{2} \mathrm{O}$ & $\frac{76.75}{76.78}$ & $\frac{5.63}{5.64}$ & $\frac{11.15}{11.19}$ & $102-104$ & 92 \\
\hline $3 f$ & $\mathrm{C}_{15} \mathrm{H}_{11} \mathrm{~N}_{3} \mathrm{O}_{2}$ & $\frac{67.90}{67.92}$ & $\frac{4.17}{4.18}$ & $\frac{15.80}{15.84}$ & $140-142$ & 90 \\
\hline $3 g$ & $\mathrm{C}_{15} \mathrm{H}_{11} \mathrm{~N}_{3} \mathrm{O}_{2}$ & $\frac{67.89}{67.92}$ & $\frac{4.15}{4.18}$ & $\frac{15.79}{15.84}$ & $130-132$ & 93 \\
\hline $3 \mathbf{h}$ & $\mathrm{C}_{17} \mathrm{H}_{16} \mathrm{~N}_{2} \mathrm{O}_{2}$ & $\frac{72.82}{72.84}$ & $\frac{5.73}{5.75}$ & $\frac{9.95}{9.99}$ & $142-144$ & 95 \\
\hline
\end{tabular}




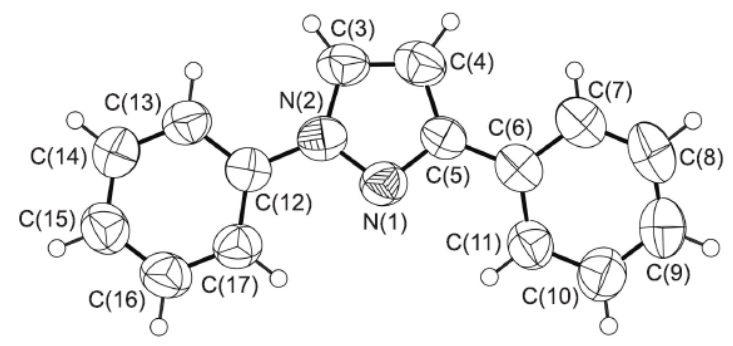

Fig 1. Crystal structure of compound 3a. Thermal ellipsoids are shown at the 50\% probability level.

The spectra of the synthesized compounds are in good agreement with their proposed structures (Table 2). The IR spectrum of 1,3-diphenyl-4,5-dihydro- $1 H$-pyrazole-5-carbonitrile (2a) shows a sharp absorption band in its IR spectrum at $2200-2210 \mathrm{~cm}^{-1}$ corresponding to $\mathrm{C} \equiv \mathrm{N}$ stretching frequency [34]. By contrast, in the IR spectra of 1,3-diphenyl-1H-pyrazole derivatives 3a-h this absorption band was absent. In addition to this, a single crystal X-ray structural study further confirms the structure of compound 3a (Fig.).

In conclusion, we have developed a new general method for the synthesis of substituted pyrazoles from easily accessible starting materials at ambient temperature using PTC. The driving force for the formation of 1,3-diphenyl-1 $H$-pyrazole may be due to the attainment of aromaticity. Hence we have been able to introduce an efficient and easy approach for the synthesis of 1,3-diphenyl-1H-pyrazole.

\section{EXPERIMENTAL}

IR spectra were recorded in nujol on an FT-IR Shimadzu 8300 spectrometer. ${ }^{1} \mathrm{H}$ NMR spectra were recorded on a Bruker Avance III spectrometer $\left(400 \mathrm{MHz}\right.$ ) in DMSO-d $\mathrm{d}_{6}$. Chemical shifts were recorded in parts per million downfield from tetramethylsilane. Mass spectra (EI, $70 \mathrm{eV}$ ) were obtained on a VG70-70H mass spectrometer. Melting points were determined on a Thomas Hoover capillary melting point apparatus with a digital thermometer. Elemental analysis was carried out on an Elementar Vairo EL instrument. TLC was performed on aluminum-packed silica plates with visualization by UV light. Chemicals were purchased from Aldrich Chemical Co.

1,3-Diphenyl-1H-pyrazole (3a). 1,3-Diphenyl-4,5-dihydro- $1 H$-pyrazole-5-carbonitrile (2a) (1.00 g, 4.0 $\mathrm{mmol})$ was taken up in THF $(20 \mathrm{ml})$ and stirred at room temperature for $10 \mathrm{~min}$. Potassium hydroxide $(0.22 \mathrm{~g}$, $4.0 \mathrm{mmol})$ and TBAB $(0.12 \mathrm{~g}, 0.4 \mathrm{mmol})$ were added to the above reaction mass and the whole stirred at room temperature for about 4-6 h. After the completion of the reaction (a single spot was observed when monitored by TLC), the reaction mixture was concentrated under reduced pressure to evaporate THF and later extracted with diethyl ether $(3 \times 20 \mathrm{ml})$, washed with water $(3 \times 20 \mathrm{ml})$. The organic extract was dried with anhydrous sodium sulfate, concentrated under reduced pressure, and crystallized to provide the pure desired compound 3a. Yield $0.84 \mathrm{~g}(95 \%)$. The product is then recrystallized from a saturated solution of methanol to obtain single crystal suitable for X-ray analysis. IR spectrum, $v, \mathrm{~cm}^{-1}: 1620(\mathrm{C}=\mathrm{N}), 1608(\mathrm{C}=\mathrm{C})$. Mass spectrum, $\mathrm{m} / \mathrm{z}$ $\left(I_{\text {rel, }} \%\right): 221[\mathrm{M}+\mathrm{H}]^{+}(100)$.

3-(4-Chlorophenyl)-1-phenyl-1H-pyrazole (3b) was obtained analogously from 3-(4-chlorophenyl)1-phenyl-4,5-dihydro-1H-pyrazole-5-carbonitrile (2b) $(1.00 \mathrm{~g}$, $3.55 \mathrm{mmol})$, potassium hydroxide $(0.20 \mathrm{~g}$, $3.55 \mathrm{mmol})$, and TBAB $(0.11 \mathrm{~g}, 0.35 \mathrm{mmol})$ as a white solid. Yield $0.84 \mathrm{~g}(94 \%)$. IR spectrum, $v, \mathrm{~cm}^{-1}: 1615$ $(\mathrm{C}=\mathrm{N}), 1610(\mathrm{C}=\mathrm{C})$. Mass spectrum, $m / z\left(I_{\mathrm{rel}}, \%\right): 254[\mathrm{M}+\mathrm{H}]^{+}(100)$.

1-Phenyl-3-(p-tolyl)-1H-pyrazole (3c) was obtained analogously from 1-phenyl-3-( $p$-tolyl)-4,5-dihydro-1H-pyrazole-5-carbonitrile (2c) $(1.00 \mathrm{~g}, 3.83 \mathrm{mmol})$, potassium hydroxide $(0.20 \mathrm{~g}, 3.83 \mathrm{mmol})$, and TBAB $(0.12 \mathrm{~g}, 0.38 \mathrm{mmol})$ as a white solid. Yield $0.79 \mathrm{~g}(88 \%)$. IR spectrum, $v, \mathrm{~cm}^{-1}: 1617(\mathrm{C}=\mathrm{N}), 1612(\mathrm{C}=\mathrm{C})$. Mass spectrum, $m / z\left(I_{\text {rel }}, \%\right): 235[\mathrm{M}+\mathrm{H}]^{+}(100)$. 
TABLE 2. ${ }^{1}$ H NMR Spectra of Compounds 3a-h

\begin{tabular}{|c|c|}
\hline $\begin{array}{l}\text { Com- } \\
\text { pound }\end{array}$ & Chemical shifts, $\delta, \operatorname{ppm}(J, \mathrm{~Hz})$ \\
\hline $3 \mathbf{a}$ & $\begin{array}{l}8.59(1 \mathrm{H}, \mathrm{d}, J=4.0, \mathrm{H}-4) ; 8.02-7.91\left(4 \mathrm{H}, \mathrm{m}, \mathrm{H}-2^{\prime}, 3^{\prime}, 5^{\prime}, 6^{\prime}\right) \\
7.66-7.44\left(4 \mathrm{H}, \mathrm{m}, \mathrm{H}-2^{\prime \prime}, 3^{\prime \prime}, 5^{\prime}, 6^{\prime \prime}\right) ; 7.39-7.19\left(2 \mathrm{H}, \mathrm{m}, \mathrm{H}-4^{\prime}, 4 "\right) ; 7.05(1 \mathrm{H}, \mathrm{d}, J=4.0, \mathrm{H}-5)\end{array}$ \\
\hline $3 \mathbf{b}$ & $\begin{array}{l}8.62(1 \mathrm{H}, \mathrm{d}, J=4.0, \mathrm{H}-4) ; 8.03-7.90\left(4 \mathrm{H}, \mathrm{m}, \mathrm{H}-2^{\prime}, 3^{\prime}, 5^{\prime}, 6^{\prime}\right) ; \\
7.62-7.51\left(4 \mathrm{H}, \mathrm{m}, \mathrm{H}-2^{\prime \prime}, 3^{\prime \prime}, 5^{\prime}, 6^{\prime \prime}\right) ; 7.05(1 \mathrm{H}, \mathrm{d}, J=4.0, \mathrm{H}-5) ; 7.34(1 \mathrm{H}, \mathrm{t}, J=8.0, \mathrm{H}-4 ")\end{array}$ \\
\hline $3 \mathbf{c}$ & $\begin{array}{l}8.52(1 \mathrm{H}, \mathrm{d}, J=4.0, \mathrm{H}-4) ; 7.97-7.92\left(4 \mathrm{H}, \mathrm{m}, \mathrm{H}-2^{\prime}, 3^{\prime}, 5^{\prime}, 6^{\prime}\right) ; \\
7.50-7.42\left(4 \mathrm{H}, \mathrm{m}, \mathrm{H}-2^{2}, 3^{\prime \prime}, 5^{\prime \prime}, 6^{\prime}\right) ; 7.05(1 \mathrm{H}, \mathrm{d}, J=4.0, \mathrm{H}-5) ; 7.30\left(1 \mathrm{H}, \mathrm{t}, J=8.0, \mathrm{H}-4^{\prime \prime}\right) ; \\
2.33\left(3 \mathrm{H}, \mathrm{s}, 4^{\prime}-\mathrm{CH}_{3}\right)\end{array}$ \\
\hline 3d & $\begin{array}{l}8.70(1 \mathrm{H}, \mathrm{d}, J=4.0, \mathrm{H}-4) ; 7.95-7.90\left(4 \mathrm{H}, \mathrm{m}, \mathrm{H}-3^{\prime}, 4^{\prime}, 5^{\prime}, 6^{\prime}\right) ; \\
7.45-7.43\left(4 \mathrm{H}, \mathrm{m}, \mathrm{H}-2^{\prime \prime}, 3^{\prime \prime}, 5^{\prime \prime}, 6^{\prime \prime}\right) ; 7.05(1 \mathrm{H}, \mathrm{d}, J=4.0, \mathrm{H}-5) ; 7.45(1 \mathrm{H}, \mathrm{t}, J=8.0, \mathrm{H}-4 ")\end{array}$ \\
\hline $3 \mathbf{e}$ & $\begin{array}{l}8.63(1 \mathrm{H}, \mathrm{d}, J=4.0, \mathrm{H}-4) ; 7.99-7.86\left(4 \mathrm{H}, \mathrm{m}, \mathrm{H}-2^{\prime}, 3^{\prime}, 5^{\prime}, 6^{\prime}\right) ; 7.44-7.40\left(4 \mathrm{H}, \mathrm{m}, \mathrm{H}-2^{\prime \prime}, 3^{\prime \prime}, 5^{\prime \prime}, 6^{\prime \prime}\right) \\
7.47\left(1 \mathrm{H}, \mathrm{t}, J=8.0, \mathrm{H}-4^{\prime \prime}\right) ; 7.04(1 \mathrm{H}, \mathrm{d}, J=4.0, \mathrm{H}-5) ; 3.90\left(3 \mathrm{H}, \mathrm{s}, 4^{\prime}-\mathrm{OCH}_{3}\right)\end{array}$ \\
\hline $3 f$ & $\begin{array}{l}8.65(1 \mathrm{H}, \mathrm{d}, J=4.0, \mathrm{H}-4) ; 7.96-7.89\left(4 \mathrm{H}, \mathrm{m}, \mathrm{H}-2^{\prime}, 3^{\prime}, 5^{\prime}, 6^{\prime}\right) ; 7.46-7.44\left(4 \mathrm{H}, \mathrm{m}, \mathrm{H}-2^{\prime \prime}, 3^{\prime \prime}, 5^{\prime \prime}, 6^{\prime \prime}\right) ; \\
7.03(1 \mathrm{H}, \mathrm{d}, J=4.0, \mathrm{H}-5) ; 7.45\left(1 \mathrm{H}, \mathrm{t}, J=8.0, \mathrm{H}-4^{\prime \prime}\right)\end{array}$ \\
\hline $3 g$ & $\begin{array}{l}8.55(1 \mathrm{H}, \mathrm{d}, J=4.0, \mathrm{H}-4) ; 7.85-7.80\left(4 \mathrm{H}, \mathrm{m}, \mathrm{H}-3^{\prime}, 4^{\prime}, 5^{\prime}, 6^{\prime}\right) \\
7.50-7.45\left(4 \mathrm{H}, \mathrm{m}, \mathrm{H}-2^{\prime \prime}, 3^{\prime \prime}, 5^{\prime \prime}, 6^{\prime \prime}\right) ; 7.20(1 \mathrm{H}, \mathrm{d}, J=4.0, \mathrm{H}-5) ; 7.40\left(1 \mathrm{H}, \mathrm{t}, J=8.0, \mathrm{H}-4^{\prime \prime}\right)\end{array}$ \\
\hline $3 \mathbf{h}$ & $\begin{array}{l}8.58(1 \mathrm{H}, \mathrm{d}, J=4.0, \mathrm{H}-4) ; 7.94-7.88\left(3 \mathrm{H}, \mathrm{m}, \mathrm{H}-2^{\prime}, 5^{\prime}, 6^{\prime}\right) ; 7.44-7.41\left(4 \mathrm{H}, \mathrm{m}, \mathrm{H}-2^{\prime \prime}, 3^{\prime \prime}, 5^{\prime \prime}, 6^{\prime \prime}\right) ; \\
7.05(1 \mathrm{H}, \mathrm{d}, J=4.0, \mathrm{H}-5) ; 7.45\left(1 \mathrm{H}, \mathrm{t}, J=8.0, \mathrm{H}-4^{\prime \prime}\right) ; 3.50\left(6 \mathrm{H}, \mathrm{s}, 3^{\prime}, 4^{\prime}-\mathrm{OCH}_{3}\right)\end{array}$ \\
\hline
\end{tabular}

3-(2-Chlorophenyl)-1-phenyl-1H-pyrazole (3d) was obtained analogously from 3-(2-chlorophenyl)1-phenyl-4,5-dihydro-1H-pyrazole-5 carbonitrile (2d) $(1.00 \mathrm{~g}, 3.55 \mathrm{mmol})$, potassium hydroxide $(0.20 \mathrm{~g}$, $3.55 \mathrm{mmol})$, and TBAB $(0.11 \mathrm{~g}, 0.35 \mathrm{mmol})$ as a white solid. Yield $0.81 \mathrm{~g}(90 \%)$. IR spectrum, $\mathrm{v}, \mathrm{cm}^{-1}: 1621$ $(\mathrm{C}=\mathrm{N}), 1610(\mathrm{C}=\mathrm{C})$. Mass spectrum, $m / z\left(I_{\mathrm{rel}}, \%\right): 254[\mathrm{M}+\mathrm{H}]^{+}(100)$.

3-(4-Methoxyphenyl)-1-phenyl-1H-pyrazole (3e) was obtained analogously from 3-(4-methoxyphenyl)-1-phenyl-4,5-dihydro-1H-pyrazole-5-carbonitrile (2e) (1.00 g, $3.60 \mathrm{mmol})$, potassium hydroxide $(0.20 \mathrm{~g}, 3.60 \mathrm{mmol})$, and TBAB $(0.11 \mathrm{~g}, 0.36 \mathrm{mmol})$ as a white solid. Yield $0.82 \mathrm{~g}(92 \%)$. IR spectrum, $v, \mathrm{~cm}^{-1}$ : $1610(\mathrm{C}=\mathrm{N}), 1608(\mathrm{C}=\mathrm{C})$. Mass spectrum, $m / z\left(I_{\mathrm{rel}}, \%\right): 251[\mathrm{M}+\mathrm{H}]^{+}(100)$.

3-(4-Nitrophenyl)-1-phenyl-1 H-pyrazole (3f) was obtained analogously from 3-(4-nitrophenyl)-1-phenyl4,5-dihydro-1H-pyrazole-5-carbonitrile (2f) $(1.00 \mathrm{~g}, 3.42 \mathrm{mmol})$, potassium hydroxide $(0.20 \mathrm{~g}, 3.42 \mathrm{mmol})$, and TBAB $(0.11 \mathrm{~g}, 0.34 \mathrm{mmol})$ as a yellow solid. Yield $0.81 \mathrm{~g}(90 \%)$. IR spectrum, $v, \mathrm{~cm}^{-1}: 1615(\mathrm{C}=\mathrm{N}), 1610$ $(\mathrm{C}=\mathrm{C})$. Mass spectrum, $m / z\left(I_{\text {rel, }} \%\right): 266[\mathrm{M}+\mathrm{H}]^{+}(100)$.

3-(2-Nitrophenyl)-1-phenyl-1H-pyrazole (3g) was obtained analogously from 3-(2-nitrophenyl)1-phenyl-4,5-dihydro-1H-pyrazole-5-carbonitrile $(\mathbf{2 g})(1.00 \mathrm{~g}, 3.42 \mathrm{mmol})$, potassium hydroxide $(0.20 \mathrm{~g}$, $3.42 \mathrm{mmol})$, and TBAB $(0.11 \mathrm{~g}, 0.34 \mathrm{mmol})$ as a white solid. Yield $0.83 \mathrm{~g}(93 \%)$. IR spectrum, $\mathrm{v}, \mathrm{cm}^{-1}: 1625$ $(\mathrm{C}=\mathrm{N}), 1612(\mathrm{C}=\mathrm{C})$. Mass spectrum, $m / z\left(I_{\text {rel }}, \%\right): 266[\mathrm{M}+\mathrm{H}]^{+}(100)$.

3-(3,4-Dimethoxyphenyl)-1-phenyl-1H-pyrazole (3h) was obtained analogously from 3-(3,4-dimethoxyphenyl)-1-phenyl-4,5-dihydro- $1 H$-pyrazole-5-carbonitrile (2h) (1.00 g, $3.25 \mathrm{mmol})$, potassium hydroxide $(0.20 \mathrm{~g}, 3.25 \mathrm{mmol})$, and TBAB $(0.10 \mathrm{~g}, 0.32 \mathrm{mmol})$ as a white solid. Yield $0.86 \mathrm{~g}(95 \%)$. IR spectrum, $v, \mathrm{~cm}^{-1}: 1612(\mathrm{C}=\mathrm{N}), 1610(\mathrm{C}=\mathrm{C})$. Mass spectrum, $m / z\left(I_{\mathrm{rel},} \%\right): 281[\mathrm{M}+\mathrm{H}]^{+}(100)$.

X-ray Structural Analysis of Compound 3a. A single crystal $\left(\mathrm{C}_{15} \mathrm{H}_{12} \mathrm{~N}_{2}, M\right.$ 220.27), block-shaped, light-yellow color, with dimensions of $0.15 \times 0.15 \times 0.15 \mathrm{~mm}$, was chosen for X-ray diffraction studies. The data were collected on a Bruker SMART APEX II X-ray diffractometer operating at $50 \mathrm{kV}$ and $30 \mathrm{~mA}$, with graphite monochromator using MoK $\alpha$ radiation. Raw data were processed and reduced by using APEX2 and SAINT software [35]. The compound crystallizes in the monoclinic space group $P 2_{1} / c$, with cell parameters $a 5.619(2)$, $b$ 9.362(4), c 22.553(8) $\AA ; \beta$ 95.429(7) $; V$ 1181.1(8) $\AA^{3}$; the final residual factor is $R_{1} 0.0502$ for 2863 reflections. Minimum and maximum ranges for the data collection are $1.81^{\circ}$ and $28.33^{\circ}$, respectively. The structure was solved by direct methods using SHELXS-97 [36]. All non-hydrogen atoms were revealed in the first Fourier map itself. Full-matrix least squares refinement was carried out using SHELXL-97 software package [36]. Anisotropic refinement of non-hydrogen atoms was started at this stage. Subsequent refinements 
were carried out with anisotropic thermal parameters for non-hydrogen atoms and isotropic temperature factors for the hydrogen atoms, which were placed at chemically acceptable positions. Crystallographic data of compound 3a have been deposited at the Cambridge Crystallographic Data Center (deposit CCDC 883145).

Doddamani Shridevi Doddaramappa is grateful to the Rajiv Gandhi National Fellowship (DV5/130(6)/RGNF/2011-2012 dated 08-08-2011) UGC, New Delhi, for providing the necessary fund to carry out the research at University of Mysore.

\section{REFERENCES}

1. $\quad$ E. V. Dehmlow and S. S. Dehmlow, Phase Transfer Catalysis, VCH Publishers, New York (1983).

2. C. M. Starks, C. L. Liotta, and M. Halpern, Phase Transfer Catalysis, Fundamentals, Applications, and Industrial Perspectives, Chapman \& Hall, New York (1994).

3. Y. Sasson and R. Neumann, Handbook of Phase Transfer Catalysis, Chapman \& Hall, New York (1997).

4. J. Elguero, P. Goya, N. Jageroic, and A. M. S.Silva, in: O. A. Attanasi and D. Spinelli (editors), Targets in Heterocyclic Systems, Vol. 6, Italian Society of Chemistry, Roma (2002), p. 52.

5. T. D. Penning, J. J. Talley, S. R. Bertenshaw, J. S. Carter, P. W. Collins, S. Docter, M. J. Graneto, L. F. Lee, J. W. Malecha, J. M. Miyashiro, R. S. Rogers, D. J. Rogier, S. Yu, G. D. Anderson, E. G. Burton, J. N. Cogburn, S. A. Gregory, C. M. Koboldt, W. E. Perkins, K. Seibert, A. W. Veenhuizen, Y. Y. Zhang, and P. C. Isakson, J. Med. Chem., 40, 1347 (1997).

6. N. K. Terrett, A. S. Bell, D. Brown, and P. Ellis, Bioorg. Med. Chem. Lett., 6, 1819 (1996).

7. J. R. Goodell, F. Puig-Basagoiti, B. M. Forshey, P.-Y. Shi, and D. M. Ferguson, J. Med. Chem., 49, 2127 (2006).

8. F. Manna, F. Chimenti, R. Fioravanti, A. Bolasco, D. Secci, P. Chimenti, C. Ferlini, and G. Scambia, Bioorg. Med. Chem. Lett., 15, 4632 (2005).

9. A. Bekhit and T. Abdel-Aziem, Bioorg Med. Chem., 12, 1935 (2004).

10. A. Bat, K. L. Dhar, S. C. Puri, A. K. Saxena, M. Shanmugavel, and G. N. Qazi, Bioorg. Med. Chem. Lett., 15, 3177 (2005).

11. R. Sliskovic, B. D. Roth, M. W. Wilson, M. L. Hoefle, and R. S. Newton, J. Med. Chem., 33, 31 (1990).

12. K. W. Moore, K. Bonner, E. A. Jones, F. Emms, P. D. Leeson, R. Marwood, S. Patel, M. Rowley, S. Thomas, and R. W. Carling, Bioorg. Med. Chem. Lett., 9, 1285 (1999).

13. J. Elguero, in: I. Shinkai (editor), Comprehensive Heterocyclic Chemistry II, Vol. 3, Elsevier Science, Oxford (1996), p. 1.

14. K. Makino, H. S. Kim, and Y. J. Kurasawa, J. Heterocycl. Chem., 35, 489 (1998).

15. Q. Lin, D. Meloni, Y. Pan, M. Xia, J. Rodgers, S. Shepard, M. Li, L. Galya, B. Metcalf, T.-Y. Yue, P. Liu, and J. Zhou, Org. Lett., 11, 1999 (2009).

16. K. Wang, D. Xiang, J. Liu, W. Pan, and D. Dong, Org. Lett., 10, 1691 (2008).

17. C. Mateos, J. Mendiola, M. Carpintero, and J. M. Mínguez, Org. Lett., 12, 4924 (2010).

18. T. Okitsu, K. Sato, and A. Wada, Org. Lett., 12, 3506 (2010).

19. C. R. Wu, Y. S. Fang, R. C. Larock, and F. Shi, Org. Lett., 12, 2234 (2010).

20. B. S. Gerstenberger, M. R. Rauckhorst, and J. T. Starr, Org. Lett., 11, 2097 (2009).

21. S. T. Heller and S. R. Natarajan, Org. Lett., 8, 2675 (2006).

22. N. S. Zefirov, S. I. Kozhushkov, and T. S. Kuznetsova, Tetrahedron, 38, 1693 (1982).

23. O. V. Kokoreva, E. B. Averina, O. A. Ivanova, S. I. Kozhushkov, and T. S. Kuznetsova, Khim. Geterotsikl. Soedin., 906 (2001). [Chem. Heterocycl. Compd., 37, 834 (2001)].

24. M. S. M. Ahmed, K. Kobayashi, and A. Mori, Org. Lett., 7, 4487 (2005). 
25. K. Alex, A. Tillack, N. Schwarz, and M. Beller, Org. Lett., 10, 2377 (2008).

26. A. Padwa, 1,3-Dipolar Cycloaddition Chemistry, Vol. 1John Wiley \& Sons, New York (1984).

27. V. K. Aggarwal, J. de Vicente, and V. Bonnert, J. Org. Chem., 68, 5381 (2003).

28. C. F. Beam, R. M. Sandifer, R. S. Foote, C. R. Hauser, and M. Gross, Synth. Commun., 6, 5 (1976).

29. M. P. Doyle, M. R. Colsman, and L. Dorow, J. Heterocycl. Chem., 20, 943 (1983).

30. M. Farag, N. A. Kheder, and Budesinsky, Tetrahedron, 53, 9293 (1997).

31. H. F. Zohdi, N. M. Rateb, and A. O. Abdelhamid, Phosphorus, Sulfur Silicon Relat. Elem., 133, 103 (1998).

32. O. S. Attaryan, A. O. Baltayan, R. E. Sagatelyan, and K. Ts. Takmazyan, Russ. J. Gen. Chem., 78, 136 (2008).

33. I. Vogel, in: A Textbook of Practical Organic Chemistry, 5th ed., Longmans, Green Co. (Ltd.), London (1989), p. 1258.

34. K. M. Lokanatha Rai, and A. Hassner, Synth. Commun., 19, 2799 (1989).

35. Bruker, 2009. APEX2 and SAINT, Bruker AXS Inc., Madison.

36. G. M. Sheldrick, Acta Crystallogr., Sect. A: Cryst. Phys., Diffr., Theor. Gen. Crystallogr., A64, 112 (2008).

37. L. J. Farrugia, ORTEP-3, J. Appl. Crystallogr., 30, 565 (1997). 